\title{
Ulrich Mosel
}

\section{Fields, Symmetries, and Quarks}

Second, Revised and Enlarged Edition

With 30 Figures 


\section{Contents}

\section{Part I. Preliminaries}

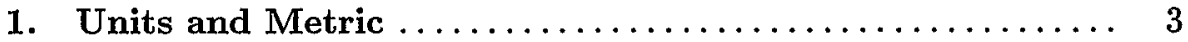

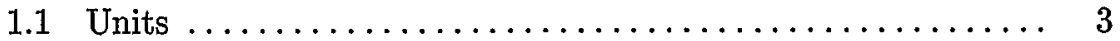

1.2 Metric and Notation $. . \ldots \ldots \ldots \ldots \ldots \ldots \ldots, 4$

Part II. Fundamentals of Field Theory

2. Classical Fields $\ldots \ldots \ldots \ldots \ldots \ldots \ldots \ldots \ldots \ldots \ldots \ldots, \quad 9$

2.1 Equations of Motion ........................ 9

2.1.1 Examples ............................ 11

2.2 Symmetries and Conservation Laws ................ 14

2.2.1 Geometrical Space-Time Symmetries ............ 15

2.2.2 Internal Symmetries . . . . . . . . . . . . . . . . . 17

3. Free Fields and Their Quantization $\ldots \ldots \ldots \ldots \ldots \ldots \ldots, 21$

3.1 Classification of Fields $\ldots \ldots \ldots \ldots \ldots \ldots \ldots \ldots \ldots \ldots, 21$

3.2 Scalar Fields . . . . . . . . . . . . . . . . . . . . . 23

3.2.1 Quantization of the Hermitian Scalar Field ......... 24

3.2.2 Quantization of the Charged Scalar Field .......... 27

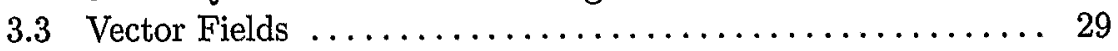

3.3.1 Massive Vector Fields ...................... 29

3.3.2 Massless Vector Fields . . . . . . . . . . . . . . . . 30

3.4 Fermion Fields $\ldots \ldots \ldots \ldots \ldots \ldots \ldots \ldots \ldots \ldots \ldots \ldots, \ldots \ldots \ldots$

3.4.1 Dirac Equation ....................... 32

3.4.2 Lagrangian for Fermion Fields . .............. 34

3.4.3 Quantization of the Dirac Field . ............. 35

3.4.4 Massless Fermions . ..................... 38

3.4.5 Neutrinos ......................... 40

3.5 Transition Rates in Quantum Field Theory ........... 44

3.6 Quantum Mechanical Consistency ................ 52 
Part III. Global Symmetries

4. Symmetries of Meson and Baryon Systems $\ldots \ldots \ldots \ldots \ldots \quad 57$

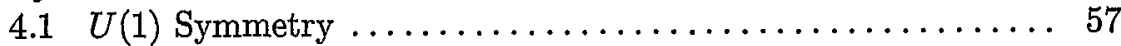

4.1.1 Properties of the Group $U(1) \ldots \ldots \ldots \ldots \ldots \ldots \ldots$

4.1.2 Structure of the Nucleon Lagrangian ............ 59

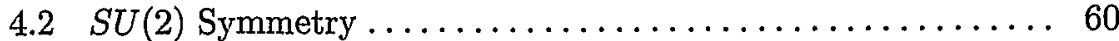

4.2.1 Properties of the Group $S U(2) \ldots \ldots \ldots \ldots \ldots \ldots, 60$

4.2 .2 General Definitions ..................... 64

4.2.3 Application to the Pion-Nucleon System .......... 64

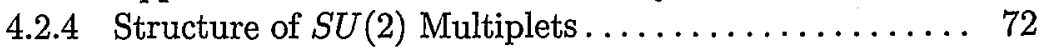

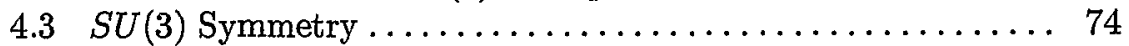

4.3.1 Properties of the Group $S U(3) \ldots \ldots \ldots \ldots \ldots \ldots \ldots$

4.3.2 Structure of $S U(3)$ Multiplets.............. 78

4.3.3 Assignments of Hadrons to $S U(3)$ Multiplets....... 83

4.3.4 $S U(3)$ Symmetry Breaking ................ 85

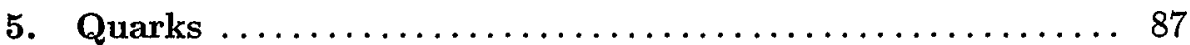

5.1 Construction of $S U(3)$ Multiplets............... 87

5.1.1 Construction of the Representation $3 \otimes \overline{3} \ldots \ldots \ldots \ldots 89$

5.1.2 Construction of the Representation $3 \otimes 3 \otimes 3 \ldots \ldots \ldots 90$

5.2 State Vectors for the Multiplets .................. 92

5.2 .1 Tensor Algebra......................... 93

5.2 .2 Hadron Multiplets....................... 96

5.3 Color Degree of Freedom ..................... 100

6. Chiral Symmetry ............................... 103

6.1 Phenomenology of $\beta$-Decay ................... 103

6.1.1 Leptonic $\beta$-Decay ........................... 103

6.1 .2 Semileptonic $\beta$-Decay ...................... 104

6.2 Current Conservation in Strong Interactions ........... 106

6.2.1 Vector Current Conservation................ 106

6.2.2 Axial Vector Current Conservation .............. 108

6.3 Chiral Symmetry Group ......................... 110

6.3.1 Chiral Symmetry Transformations for the Fermions . . . 110

6.3.2 Chiral Symmetry Transformations for the Mesons .... 112

7. Spontaneous Global Symmetry Breaking ............ 115

7.1 Goldstone Theorem .......................... 115

7.1.1 Goldstone Bosons ........................ 116

7.2 Examples of the Goldstone Mechanism .............. 119

7.2.1 Spontaneous Breaking of a Global Non-Abelian Symmetry ............. 119

$7.2 .2 \quad \sigma$-Model. .............................. 121

7.2.3 Nambu-Jona-Lasinio Model ................ 129 
Part IV. Local Gauge Symmetries

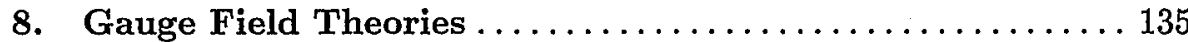

8.1 Conserved Currents in QED ..................... 135

8.2 Local Abelian Gauge Invariance .................. 137

8.3 Non-Abelian Gauge Fields . ..................... 139

8.3.1 Lagrangian for Non-Abelian Gauge Field Theories .... 139

8.3.2 Properties of Non-Abelian Gauge Field Theories ..... 144

9. Spontaneous Symmetry Breaking in Gauge Field Theories 147 9.1 Higgs Mechanism .......................... 147

9.2 Spontaneous Breaking of a Local Non-Abelian Symmetry .... 150

9.3 Summary of the Higgs Mechanism ................ 155

\section{Part V. Electroweak Interaction}

10. Weak Interactions of Quarks and Leptons ............ 159

10.1 Phenomenological Introduction .................... 159

10.1.1 Strangeness Changing Weak Decays ........... 159

10.1.2 Neutral Currents .......................... 160

10.2 Intermediate Vector Bosons ..................... 161

10.3 Fundamentals of a Theory of Weak Interactions .......... 164

11. Electroweak Interactions of Leptons . .............. 167

11.1 Leptonic Multiplets and Interactions ................ 167

11.1.1 Electroweak Currents ....................... 174

11.2 Lepton Masses ............................. 175

11.3 Electroweak Interactions $\ldots \ldots \ldots \ldots \ldots \ldots \ldots \ldots \ldots \ldots \ldots$

11.3.1 Generalization to Other Leptons .............. 180

11.4 Parameters of the Lagrangian .................... 180

11.4.1 Charged Current Experiments ................ 180

11.4.2 Neutral Current Experiments ................ 182

12. Electroweak Interactions of Quarks $\ldots \ldots \ldots \ldots \ldots \ldots \ldots 187$

12.1 Hadronic Multiplets . . . . . . . . . . . . . . . . . . . 187

12.1.1 Hadron Masses........................... 190

13. Electroweak Interactions of Quarks and Leptons ........ 193

13.1 Lagrangian of Electroweak Interactions . . . . . . . . . . 193

13.2 Standard Model .............................. 194 
14. CP Invariance of Electroweak Interactions . . . . . . . . 197

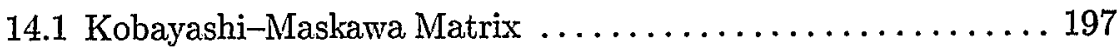

14.2 Unitarity of the KM Matrix .................... 198

$14.3 K^{0}$ Decay and CP Violation ...................... 201

14.4 CP Invariance and the KM Matrix............... 203

\section{Part VI. Strong Interaction}

15. Quantum Chromodynamics ..................... 209

15.1 Gauge Group for Strong Interactions . . . . . . . . . . . 209

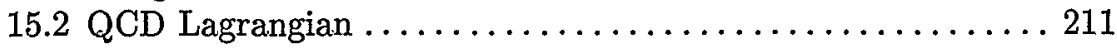

15.3 Properties of QCD ........................... 213

15.3.1 Scale Invariance ...................... 213

15.3.2 Chiral Invariance ...................... 214

15.3.3 Antishielding and Confinement.............. 215

15.3.4 Deconfinement Phase Transition............... 217

\section{Part VII. Hadron Structure}

16. Bag Models of Hadrons ......................... 223

16.1 Potential Well in the Dirac Theory ................ 223

16.2 The MIT Bag ............................ 228

16.2.1 Fermions in the MIT Bag . ................. 229

16.2.2 Hadron Masses.......................... 232

16.2.3 Gluons in the MIT Bag .................. 234

16.2.4 Hyperfine Structure of Bag States ............. 235

16.2.5 Magnetic Moments of the Nucleon ............. 243

16.2.6 Axial Vector Current .................... 246

16.2.7 Chiral Symmetry in the MIT Bag ............. 251

17. Soliton Models of Hadrons.................... 257

17.1 Skyrmion Model........................... 258

17.2 Hybrid Chiral Bag Model....................... 262

17.3 Linear $\sigma$-Model ............................ 269

17.4 Friedberg-Lee Soliton Bag Model . . . . . . . . . . . . . . 272

17.5 NJL Soliton Model ........................... 274 
Part VIII. Appendices

A. Solutions of the Free Dirac Equation $\ldots \ldots \ldots \ldots \ldots \ldots \ldots \ldots \ldots$

A.1 Properties of Free Dirac States . . . . . . . . . . . . . . . 279

A.2 Massless Fermions .......................... 284

A.3 Dirac and Majorana Fields $\ldots \ldots \ldots \ldots \ldots \ldots \ldots \ldots . \ldots \ldots 6$

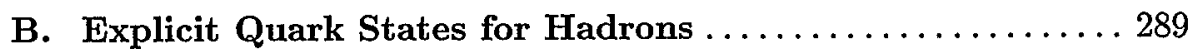

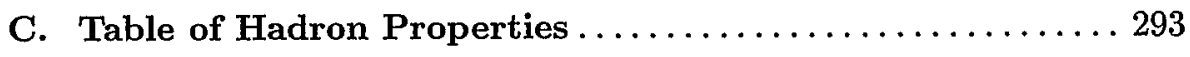

Bibliography by Subject $\ldots \ldots \ldots \ldots \ldots \ldots \ldots \ldots \ldots \ldots \ldots \ldots$

References.................................... 303

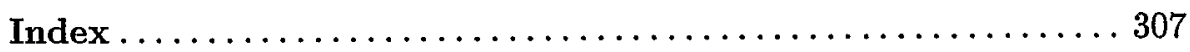

